\title{
A 12-week, whole-food carbohydrate-restricted feasibility study in overweight children
}

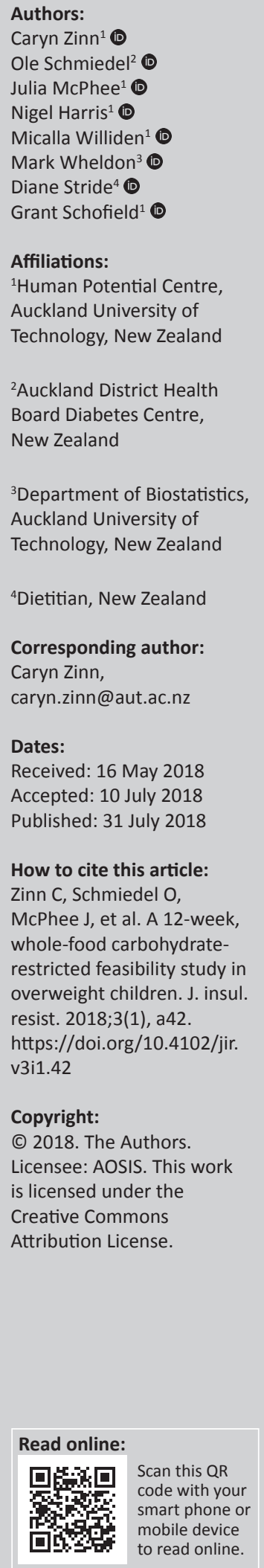

Background: Childhood obesity is a global health concern. Conventional nutrition guidelines have come under scrutiny in helping to achieve long-term healthy weight. An alternative carbohydrate-restricted, higher fat approach has shown to be effective in adults, but research is limited in youth.

Aim: To assess the feasibility of a 12-week whole-food, carbohydrate-restricted diet on weight loss and metabolic health.

Setting: Overweight children aged 8-13 years.

Methods: In this single-arm study, 25 overweight children were provided with wholefood, carbohydrate-restricted dietary guidelines. Primary outcomes - dietary acceptability, adherence and affordability - were assessed qualitatively weekly (telephone) and postintervention (focus groups). Secondary outcomes-Body mass index (BMI), waist circumference, lipids and glycaemic control measures - were assessed at 0 and 12 weeks. Change scores were analysed using the $t$-statistic and interpreted using the statistical significance threshold, $p<0.05$.

Results: Overall, dietary acceptability was mostly positive, and reports of affordability by parents were mixed. Attrition rates were high (48\%); adherence was influenced, positively and negatively, by levels of support from friends and family. Completing children reduced BMI by $2.1 \pm 1.5 \mathrm{~kg} \cdot \mathrm{m}^{2}(p<0.05)$. Key blood parameter changes included a reduction in triglycerides $(-0.17 \pm 0.48 \mathrm{mmol} / \mathrm{L} ; p=0.242)$ and an increase in high-density lipoprotein (HDL) cholesterol $(0.24 \pm 0.19 \mathrm{mmol} / \mathrm{L} ; p<0.05)$.

Conclusion: Children achieved some weight loss and health outcome success using this dietary approach. For sustainable weight loss maintenance, full family and health professional support, particularly on a more intensive level at the start, may be required.

\section{Introduction}

Childhood obesity is one of the greatest global public health challenges of our time. Over the past 30 years, it has more than doubled in children and quadrupled in adolescents worldwide. ${ }^{1}$ The New Zealand population echoes this trend, with $11 \%$ of children aged 2-12 years reported as being obese in the most recent health survey, and a further $22 \%$ overweight. ${ }^{2}$ Obese youth are at greater risk for developing comorbidities and metabolic disorders as adults, as well as experiencing psychological consequences from obesity-related stigmatisation. ${ }^{1}$

Despite the effort invested, globally, to alleviate childhood obesity, it continues to rise incessantly. ${ }^{3}$ It has been approximately 50 years since the establishment of the global standard dietary guidelines. There is currently debate surrounding the evidence upon which these guidelines are based both in terms of their general guiding principles and in relation to the specific proportion of macronutrients derived from carbohydrate and fat. ${ }^{4,5}$ Dietary guidelines are based on a moderate-to-high carbohydrate, moderate protein and low-fat approach. ${ }^{6}$

More recently, an alternate option for dietary guidance has emerged, ${ }^{7}$ which has been suggested to positively impact weight change and improve metabolic health. This carbohydrate-restricted, higher fat approach has become popular in both research and practice settings for both adults and children. ${ }^{8}$ In children, few studies exist, but on the whole, they are at least as effective as mainstream nutrition for reducing body weight and waist circumference, and appear to be more effective for improving metabolic risk factors such as triglycerides, HDL cholesterol (HDLc) and glycaemic control., ${ }^{9,10,11,12,13}$ Low carbohydrate, high-fat (LCHF) approaches have been criticised because of a lack of knowledge around adherence and affordability for families. ${ }^{14}$ We agree that 
the issues around adherence to this way of eating, along with other real-life aspects associated with sustainable behaviour change, are critical, especially for overweight or obese youth in a family setting. This requires further translational research. As such, our study assessed three key feasibility issues: diet acceptability, adherence and affordability, alongside the efficacy of a whole-food, LCHF diet on weight loss and metabolic health in overweight children.

\section{Research methods and design}

The study took place in Hawkes Bay, New Zealand. It was a single-arm trial (no control group), which aimed to determine the feasibility from several perspectives, that is diet acceptability, adherence, affordability and efficacy, of applying a 12-week whole-food LCHF intervention on overweight children aged 8-13 years and their families. The trial was approved by the Northern A Health and Disability Ethics Committee, New Zealand, No. 14/NTA/99. All caregivers provided written consent on behalf of their children and for the use of their data in the study. The trial was retrospectively registered with the Australian New Zealand Clinical Trials Registry on 28 November 2016 (Registration reference: ACTRN12616001640493).

\section{Recruitment}

Our target sample size for this feasibility study was $25 ; 28$ children were invited, and three declined to participate. Children were included if they met the criteria for being overweight according to the Centers for Disease Control (CDC) definition of $\geq 85$ th percentile for BMI-for-age, ${ }^{15}$ free from any diagnosed medical condition (i.e. diabetes, renal, heart or liver disease, thyroid or endocrine dysfunction, genetic disorder), physical or mental disability, and had not undergone weight loss treatment (diet or medication) in the previous three months. Suitable children were identified from general practitioner (GP) databases. Their caregivers were contacted by their GP, or practice nurse and those interested were sent further information and a consent form.

\section{Protocol}

All participants and their caregivers or families were invited to attend a 90-min workshop delivered by a dietitian and an endocrinologist, during which the LCHF nutrition approach was explained. It focused on a moderate level of carbohydrate restriction and was based on a whole-food approach, discouraging consumption of processed food wherever possible. Participants were not provided with an individualised calorie-controlled plan, or prescribed a macronutrient breakdown. Instead, they received a food guide (i.e. preferred foods and foods to avoid). It was anticipated that this style of eating reflected a moderate carbohydrate restriction, low glycaemic index diet, with intake approximating less than the lower range specified in mainstream New Zealand food and nutrition guidelines, that is $45 \%$ of total energy; a higher fat intake, approximating more than $33 \%$ of total energy, and a moderate protein intake, approximating $15 \%-25 \%$ of total energy. ${ }^{16}$ Foods that were encouraged included vegetables (with a focus on non-starchy vegetables), fruit, meats, eggs, full-fat dairy products, nuts and seeds, avocado and healthy oils (olive or avocado). Fruit and starchy vegetables were encouraged in limited quantities because of their high carbohydrate load. Packaged snack food, cereals and grains and foods containing refined sugar were strongly discouraged. Participants' caregivers were provided access to a website specifically designed for the study, which profiled support information including recipes, food and shopping lists and information on strategies to integrate the LCHF lifestyle into the family setting. Participants were not requested to change their levels of physical activity but rather instructed to continue with their usual activities.

Prior to starting the intervention, participants attended a dietitian's clinic in a fasted state, where they had their baseline anthropometry measures taken, and thereafter attended the hospital for their blood tests. This was repeated at the conclusion of the intervention at 12 weeks. Participants' caregivers were contacted by telephone each week to assist with any questions they might have and to assess progress using the feasibility aspects as prompting questions (see Appendix 1: Telephone protocol). At weeks 4 and 8, participants attended the clinic for anthropometry measures and a follow-up consultation. Focus groups were conducted at the end of the intervention; separate sessions were held for the participants and for their caregivers (see Appendix 2: Focus group questions).

\section{Outcome variables}

The primary outcome variables were diet acceptability, adherence and affordability, which were measured qualitatively in two ways: firstly, as part of the individual interviews with caregivers during the weekly telephone conversations. Parents were asked open-ended questions about these three aspects, along with other more general questions to assess overall progress; data were documented in written format. Secondly, as part of the focus groups conducted with parents or caregivers and children at the end of the intervention. In addition to the parent focus groups, before they commenced, we also asked each of them, individually, about the affordability of the diet, and documented this accordingly in written format. We did this to provide another, more private, opportunity to discuss the financial aspect with a member of the research team, with whom they had built rapport over the course of the study, in case they felt uncomfortable talking about their finances in a group of mixed socio-economic individuals.

Secondary outcome variables were anthropometric measures (BMI - calculated as weight $[\mathrm{kg}] /$ height $^{2}[\mathrm{~m}]$, weight, waist circumference), fasting lipid profiles (HDLc, LDL cholesterol [LDLc] and triglycerides) and glycaemic control (serum glucose, serum insulin and $\mathrm{HbA1c}$ ). Body weight was measured using weighing scales (Tanita-410, Tanita Corporation America Inc, Arlington Heights, IL), height using 
a stadiometer (Tanita HR-200, Tanita Corporation America Inc) and waist circumference using a Lufkin W606PM tape measure. BMI $z$-scores were calculated for comparison with that reference population using age- and genderspecific norms from the CDC growth charts. ${ }^{17}$ The metabolic markers were measured by venepuncture technique using laboratory serum assays. Homeostatic model assessment Insulin resistance (HOMA-IR) was used to depict insulin resistance and was calculated using the formula: (fasting insulin $(\mathrm{mU} / \mathrm{L}) \times$ fasting glucose $(\mathrm{mmol} / \mathrm{L})) / 22.5$, at baseline and at the completion of the intervention.

\section{Analysis}

Qualitative data were analysed according to standard qualitative data analysis protocols as follows. The focus groups were recorded and transcribed. All data from the focus groups and from the weekly interviews were uploaded into qualitative data analysis computer software (NVIVO 11, QSR International, Victoria, Australia) and analysed using thematic analysis. We examined the transcripts for emerging themes guided by the three categories; diet acceptability, adherence and affordability. Data were coded by and discussed between two members of the research team, and are presented in tabular format as key themes with supporting transcripts. The data collected, analysed and presented for the qualitative aspect of the study represent that of both the non-completers (from the telephone conversations prior to drop out) and the completers (those that attended the focus groups) to avoid any potential bias of presenting data from only the most motivated participants.

All secondary outcomes of participants (completers only) are presented as means and standard deviations for the preand post-measures. Because of the explorative nature of this study and our small sample size, quantitative data are presented as individual responses. We elected to apply a probability statistic using a paired $t$-test to determine the statistical meaning of the change from baseline for key indicators, where $p<0.05$ was considered significant. The Holm-Bonferroni method was used to compute adjusted $p$-values that accounted for simultaneously testing multiple hypotheses. ${ }^{18}$ Cohen's effect size was used to describe the magnitude of change. These were calculated using the change score (i.e. post minus baseline) divided by the standard deviation of the baseline value and the following thresholds were used to describe the magnitude of each result: small (0.2), moderate (0.5) and large (0.8). We acknowledge that applying statistical models hold limited meaning in this context and it is not our intention to make any inferences about these outcomes to overweight child populations. As such, outcomes with a significance level of $p<0.05$ should be considered a trend only; and significant only in the sense that further work is required to substantiate these findings.

\section{Results}

A total of 25 children were recruited into the study, and 13 of them completed (attrition was $48 \%$ ). Table 1 presents the
TABLE 1: Baseline participant characteristics and blood marker variables.

\begin{tabular}{|c|c|}
\hline Participant characteristics & Total \\
\hline \multicolumn{2}{|l|}{ Gender ( $\boldsymbol{n}[\%])$} \\
\hline Female & $15(60)$ \\
\hline Male & $10(40)$ \\
\hline \multicolumn{2}{|l|}{ Ethnicity ( $[$ [\%]) } \\
\hline New Zealand or other European & $11(44)$ \\
\hline Maori & $12(48)$ \\
\hline Pacific Island & $2(8)$ \\
\hline Age, mean \pm SD (years) & $10.52 \pm 1.66$ \\
\hline Variables & Mean \pm SD \\
\hline \multicolumn{2}{|l|}{ Weight (kg) } \\
\hline All & $68.4 \pm 16.6$ \\
\hline Female & $65.9 \pm 17.0$ \\
\hline Male & $72.0 \pm 16.2$ \\
\hline \multicolumn{2}{|l|}{ Height (cm) } \\
\hline All & $150.6 \pm 9.6$ \\
\hline Female & $148.5 \pm 9.7$ \\
\hline Male & $153.8 \pm 8.9$ \\
\hline \multicolumn{2}{|l|}{ BMI (kg.m²) } \\
\hline All & $29.7 \pm 4.1$ \\
\hline Female & $29.4 \pm 4.3$ \\
\hline Male & $30.1 \pm 4.0$ \\
\hline \multicolumn{2}{|l|}{ BMI z-score } \\
\hline All & 2.2 \\
\hline Female & 2.2 \\
\hline Male & 2.4 \\
\hline \multicolumn{2}{|l|}{ Waist (cm) } \\
\hline All & $100.1 \pm 9.5$ \\
\hline Female & $98.2 \pm 7.7$ \\
\hline Male & $103.0 \pm 11.7$ \\
\hline Waist:height & $0.7 \pm 0.1$ \\
\hline $\mathrm{HDLc}(\mathrm{mmol} / \mathrm{L})$ & $0.8 \pm 0.4$ \\
\hline LDLc (mmol/L) & $1.5 \pm 0.5$ \\
\hline Triglyceride (mmol/L) & $0.9 \pm 0.5$ \\
\hline Triglyceride:HDLc & $1.3 \pm 0.8$ \\
\hline $\mathrm{HbA} 1 \mathrm{C}(\mathrm{mmol} / \mathrm{mol})$ & $29.9 \pm 2.5$ \\
\hline Glucose (mmol/L) & $4.4 \pm 0.9$ \\
\hline Insulin (pmol/L) & $106.6 \pm 86.9$ \\
\hline HOMA-IR & $3.27 \pm 3.5$ \\
\hline
\end{tabular}

SD, standard deviation; HOMA-IR, Homeostatic model assessment - Insulin resistance; HDL, high-density lipoprotein; LDL, low-density lipoprotein.

baseline participant demographics. While all children were classified as overweight, their mean markers of metabolic health were all within their respective healthy range apart from HDLc, which was just below the lower end of the healthy range for children. ${ }^{19}$

Table 2 presents the key themes discovered under the broad feasibility aspects of dietary acceptability, adherence and affordability, and their respective supporting transcripts. Overall, food was considered acceptable to both the children and their families for the most part. All participants were able to describe particular foods that they had enjoyed, which were not part of their previous food intake. Social support and norms were key variables associated with adherence for children and parents. The affordability of the LCHF dietary approach varied between families.

Table 3 provides a summary of the pre- and post-intervention outcomes for each of the secondary variables for completing 
TABLE 2: Themes and supporting transcripts from caregivers and children.

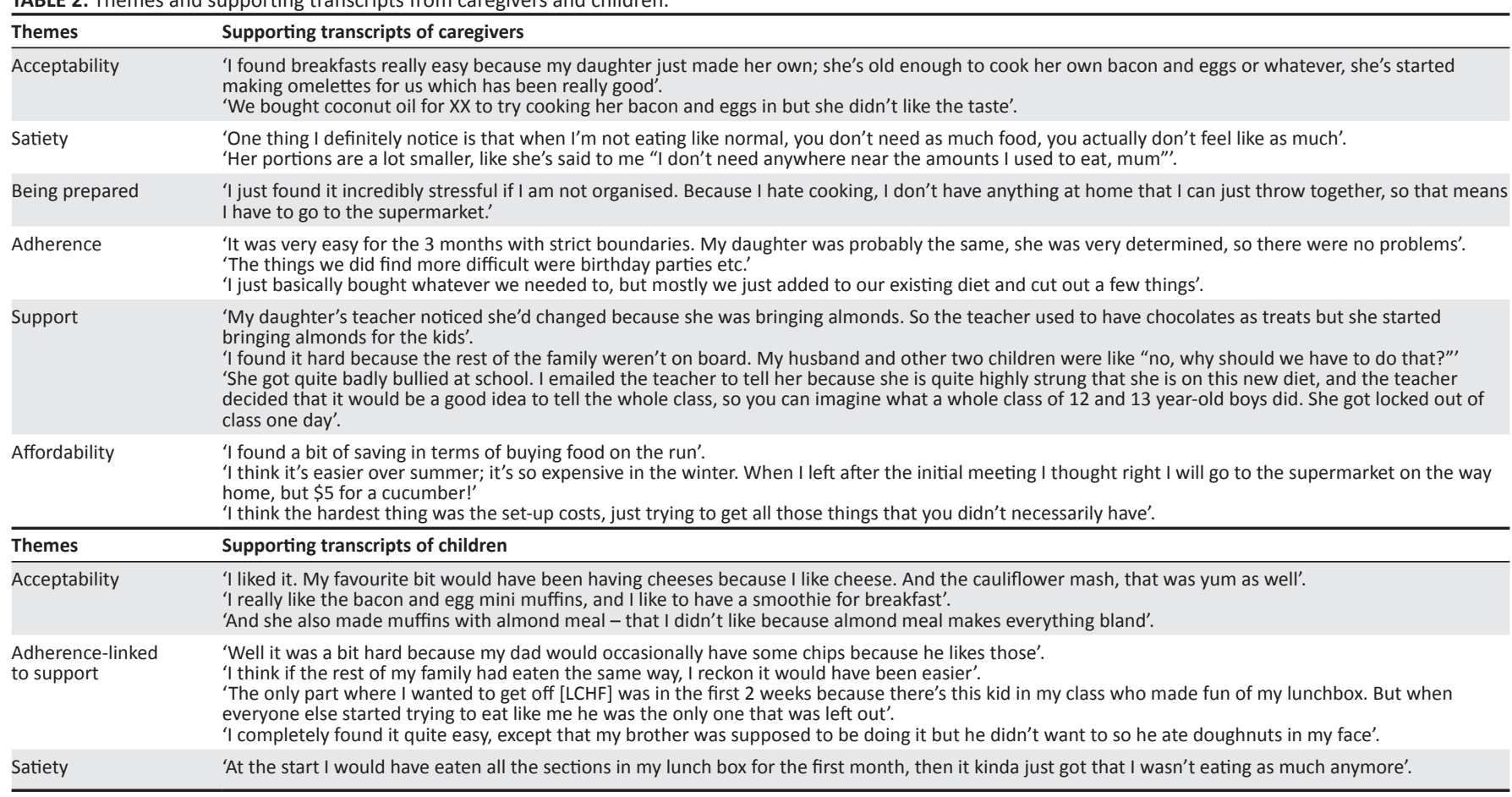

TABLE 3: Change in metabolic health markers of completing participants.

\begin{tabular}{lcccc}
\hline Variables & $\begin{array}{c}\text { Baseline } \\
\text { Mean } \pm \text { SD }\end{array}$ & $\begin{array}{c}\text { Week 12 } \\
\text { Mean } \pm \text { SD }\end{array}$ & $p$-value & Effect size \\
\hline Weight (kg) & $62.9 \pm 10.7$ & $59.7 \pm 12.4$ & $p=0.006^{*}$ & -0.3 \\
BMI (kg.m²) & $28.1 \pm 3.1$ & $26.0 \pm 3.8$ & $p=0.0003^{*}$ & -0.7 \\
BMI z-score & 2.2 & 1.9 & - & - \\
Waist (cm) & $97.8 \pm 8.5$ & $92.5 \pm 9.9$ & $p=0.0007^{*}$ & -0.8 \\
Waist:height & $0.7 \pm 0.1$ & $0.6 \pm 0.1$ & $p=0.0003^{*}$ & -0.9 \\
Glucose (mmol/L) & $4.6 \pm 0.9$ & $4.8 \pm 0.4$ & $p=0.521$ & 0.2 \\
Insulin (pmol/L) & $119.0 \pm 89.0$ & $85.0 \pm 52.6$ & $p=0.179$ & -0.4 \\
HbA1C (mmol/mol) & $30.4 \pm 2.9$ & $31.0 \pm 2.0$ & $p=0.286$ & 0.2 \\
HOMA-IR & $3.9 \pm 4.2$ & $2.6 \pm 1.7$ & $p=0.273$ & -0.3 \\
LDLc (mmol/L) & $1.6 \pm 0.5$ & $2.0 \pm 0.6$ & $p=0.048^{*}$ & 0.8 \\
HDLc (mmol/L) & $0.7 \pm 0.2$ & $0.9 \pm 0.2$ & $p=0.0007^{*}$ & 1.5 \\
Triglycerides (mmol/L) & $1.0 \pm 0.5$ & $0.8 \pm 0.2$ & $p=0.242$ & -0.3 \\
Triglyceride:HDLc & $1.5 \pm 0.8$ & $0.9 \pm 0.5$ & $p=0.039 *$ & 0.7 \\
\hline
\end{tabular}

HDL, High-density lipoprotein; LDL, low-density lipoprotein; HOMA-IR, HOMEostatic model assessment - Insulin resistance; SD, standard deviation; *, Significant finding.

participants, their respective means and standard deviations, and associated $p$-values for the change scores. Statistical significance was obtained for the majority of the variables with effect sizes ranging between small and large. Of note was HDLc, which showed the largest effect. Figure 1 shows individual responses for several of these variables. All participants apart from one trended downwards for BMI and all trended downwards for waist:height. Fasting glucose and insulin trended upwards and downwards, respectively; one particular participant had a substantial decrease in fasting glucose, and two participants in fasting insulin. The LDLc trend was mixed with two participants experiencing a rise above the reference range. Triglycerides trended downwards with two participants experiencing a substantial drop in levels. All participants experienced an increase in HDLc.

\section{Discussion}

This is the first study to qualitatively investigate the feasibility of a whole-food, LCHF nutrition approach for weight loss in children. Overall, the findings suggest that there is potential, particularly with support from health professionals, friends and family as these factors impact adherence and ultimate success.

Parents and children found the majority of the food choices to be acceptable. Not every food or meal suggested was enjoyed; however, it is likely that whatever the nutrition paradigm, children have unique food likes and dislikes with 'fussy eating' being a relatively common phenomenon during childhood in general. ${ }^{20}$ Despite no formal diet diary comparison of previous versus current food choices, parents and children reported consuming more fruit, vegetables, nuts, cheese, cauliflower dishes and eggs than before.

There was mention that children became bored with the lack of variety and the restrictions put on food choices, particularly towards the end of the intervention. Again, while this might not necessarily be different from boredom experienced with usual food intake, it does emphasise the need for more support in this area. Despite a plethora of resources on the Internet, it might be that a structured, comprehensive package of meal ideas and recipes alongside some practical cooking class guidance is required to better support families and to help prevent boredom. Kirk et al. attributed their long-term weight loss success in all three dietary intervention groups, to the initial intensity of the guidance applied. ${ }^{11}$

Adherence was directly related to the level of social support. Both parents and children reported LCHF eating easy to 

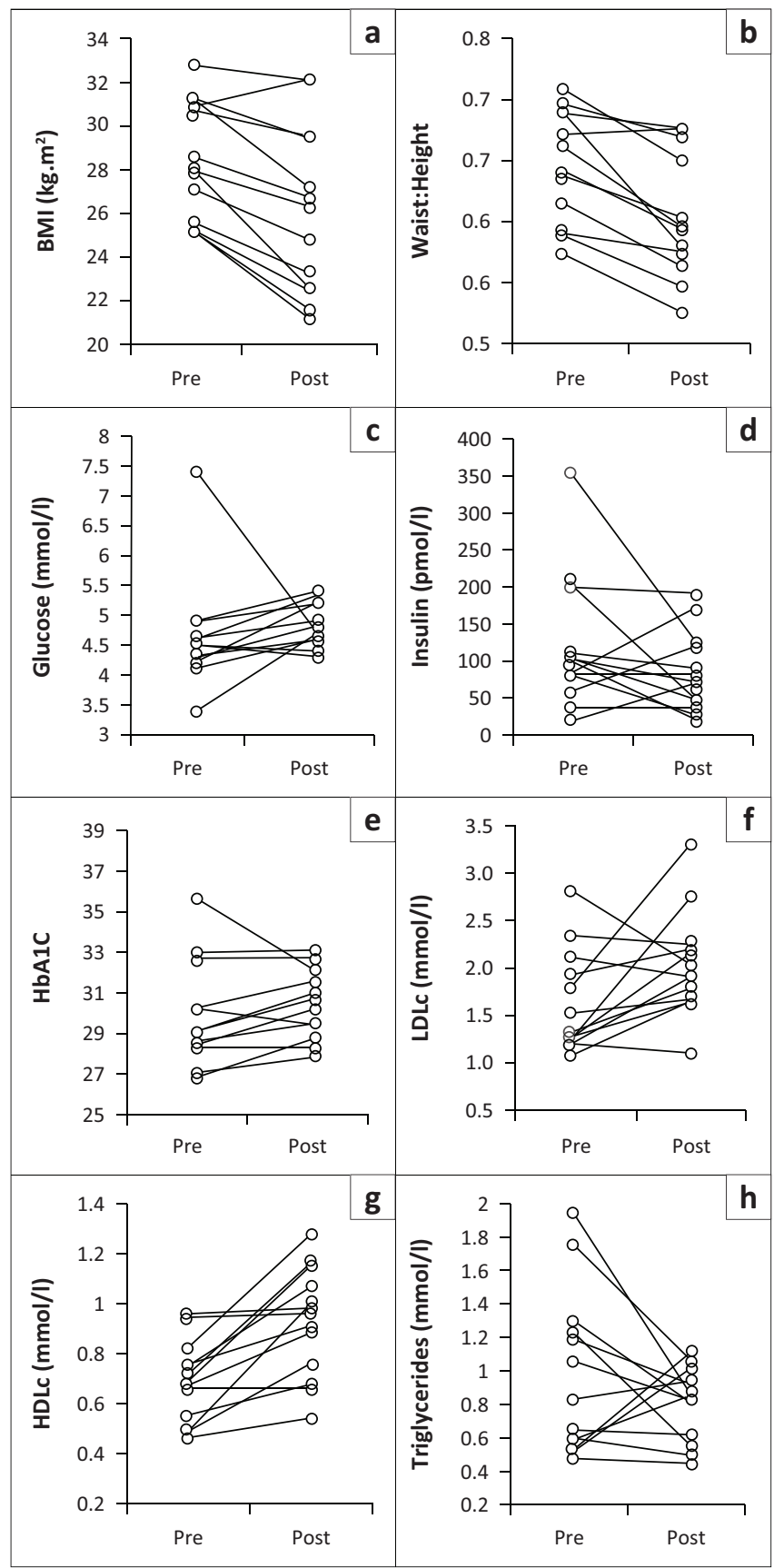

FIGURE 1: Individual responses for anthropometric and metabolic health outcome variables.

adhere to; however, when friends and family members were not supportive, adherence was challenged and often compromised. Children expressed that with family support, LCHF eating would not only have been easier to adhere to but also to continue with. Some mentioned that when friends and acquaintances understood why they were eating this way, they became supportive and even aligned their food choices. Parents also attributed their children's success to their determination and willingness to participate. Bailes et al. describe two children reporting difficulty complying with their low carbohydrate diet at school as the reason for not completing their 8-week intervention. While the theme of support is similar to our study, in this case, the diet was extreme $(<30 \mathrm{~g}$ carbohydrate per day), ${ }^{21}$ and therefore the value of study comparison is limited.
We propose that the satiety that usually accompanies LCHF diets may have an important influence on adherence, which ultimately affects sustainability and success. Research evidence suggests that protein is the most satiating macronutrient. ${ }^{22,23}$ While not intended to be high in protein, it is likely that protein levels were raised, inadvertently, because of the nature of foods being consumed with more freedom than usual, that is cheese, nuts and eggs. Regular communication with participants' caregivers verified this notion.

In the only systematic review of weight loss randomised controlled trials in children comparing diets with differing macronutrient profiles, authors refer to a low carbohydrate diet as being hard to adhere to long-term because of restriction of foods. ${ }^{14}$ It could be contested that there is as much dietary restriction that accompanies low-fat dieting, a point that is corroborated in Sondike et al.'s findings, who reported noncompliance in their low-fat group of adolescents because of limited food choice, alongside hunger and an unpalatable diet. ${ }^{13}$ These researchers suggest that a carbohydraterestricted diet may be easier for adolescents to follow than a low-fat diet.

Adherence was also assessed quantitatively by anthropometric measures. One cannot assume that weight loss was achieved as a direct result of adherence to LCHF eating; however, we are confident in saying that adherence was good in these children, as a dietary history (24-h dietary recall and food frequency questionnaire) was measured during weekly phone calls and during the two faceto-face consultations. The data for these measures are not presented here as they were incomplete; researchers found inconsistencies with availability of caregivers, many of which did not have voicemail services on their telephones or did not return calls. The attrition rate was considered high in this study (48\%). It was assumed that adherence was compromised in the non-completers, apart from two families, who despite documented weight loss progress for the first 8 weeks could not logistically get to their final set of measures. Despite our best efforts, we were unable to contact these families to establish reasons for discontinuation. High attrition rates are not uncommon in weight loss studies in general, and our rate is consistent with those reported in similar studies, with both LCHF $(22 \%-70 \%)$ and low-fat approaches $(0 \%-70 \%)$ showing wide variation. ${ }^{9,12,13,21}$ While no clear pattern emerges as to the attributed reasons for high attrition, it might be worth noting that Demol et al. report $0 \%$ attrition in their group of 55 adolescents. Theirs was a highly intensive intervention that included weekly detailed menu plans and dietetic and psychology support, ${ }^{9}$ which might have contributed to this outcome. The issue of attrition in weight loss studies, in general, warrants further investigation, largely to assess whether it is attributed more to the actual diet, the intensity of the intervention, the study burden or a combination of these factors.

Mixed reports of affordability included cost savings when family members supported LCHF eating because of 
alternative foods not being purchased, and extra costs spent if these foods were extra purchase items. Some families reported vegetables being expensive during some months. This, however, would not differ from mainstream guidance, where a high intake of vegetables is also advocated. Most parents reported that new foods added extra cost initially, but then savings were made on foods that were no longer being purchased. Some commented that the cost increase was outweighed by the benefits and was not a barrier to sustainability. These findings indicate that further work emphasising lower cost items, LCHF budgeting strategies and incorporating cost data would be valuable.

Our study demonstrated significant improvements in BMI and waist:height ratio at three months. We do not know whether this would be sustainable long-term; however, even with this short intervention, we were able to demonstrate the significant weight loss potential of this dietary approach. This finding, along with the significant finding of improved HDLc, compares favourably with that of similar studies. ${ }^{9,10,11,12,13,21}$ We were unable to confirm a significant mean change in insulin resistance, using HOMA-IR. However, at baseline, six children exceeded the threshold confirming insulin resistance (HOMA-IR $>3.16$ ) ${ }^{24}$ and these participants showed the most meaningful reduction at three months. Yajnik and colleagues measured prepubertal glucose and insulin metabolism via HOMA-IR, and assessed cardiovascular risk profiles at age 21 in 357 participants. Those in the highest HOMA-IR quartile had a more adverse cardiovascular risk profile compared with those in the lowest quartile, suggesting that interventions to improve glucose and insulin metabolism in childhood may reduce cardiovascular risk in adulthood. ${ }^{25}$

Two children in our study increased LDLc above the reference range, a finding that has been reported in similar studies. ${ }^{11,13}$ Despite this, one of these children showed improvements in all other parameters. The triglyceride:HDLc ratio is becoming increasingly well recognised and utilised in a clinical setting as a useful predictor of insulin resistance, ${ }_{r}^{26,27,28}$ we demonstrated a statistically significant change in this variable in our sample. In Pacifico et al.'s sample of 548, overweight and obese children aged 6-16 years, a high triglyceride:HDLc ratio was associated with increased carotid artery intima-media thickness (cIMT) ${ }^{29}$ Authors suggested that a high triglyceride:HDLc ratio may help to identify children who already have an increased risk of metabolic and vascular changes. In our sample, it could be that in this metabolic context, the benefits from an improved cluster of risk factors impacting chronic disease may outweigh any impact from a raised LDLc. There is current debate surrounding the LDLc fraction and its overall effect on disease risk; this warrants further research in the context of LCHF diet. ${ }^{30,31,32,33}$

Our study has several limitations. It was a short-term study with a small sample, no control group and was not controlled for exercise. In particular, the lack of a control group prevents us from drawing causal inferences. We also do not have a comprehensive set of diet data to objectively verify adherence to this way of eating over the 12 weeks. A further limitation of the study is using HOMA-IR to determine insulin resistance. We acknowledge the emerging evidence, showing that insulin resistance and the use of fasting insulin measures to determine insulin resistance may no longer be the best methods to determine disease risk. ${ }^{34}$ However, these measures were considered appropriate in the context of the key study objectives. Despite these limitations, we demonstrated that using a moderate LCHF approach, even in this short time frame, improved indicators of disease risk can be experienced. A larger and more extended study is warranted to show sustained improvement in these metabolic outcomes.

Key strengths of this work were that it profiled a real-life translation of a carbohydrate-restricted style of eating amongst a mixed ethnic group of families with overweight children. The qualitative exploration into how both children and their families fared when adopting an LCHF way of eating is a further strength as this type of information provides us with valuable insights from both caregivers' and children's perspectives as to how best to move forward with the application of LCHF nutrition, in research and in practice. We would recommend that future work of this nature should progress this by capturing qualitative outcomes in addition to establishing dietary efficacy. Finally, an additional strength of this work was the range of health professional expertise and their input in this study, from recruitment through to study implementation, that is, GPs, an endocrinologist, two registered dietitians and a registered nutritionist.

\section{Conclusion}

Our study was primarily a feasibility study to investigate the translation of the LCHF dietary approach in the homes and lives of children and their families. As such, we are encouraged by the favourable outcomes and the stories of influence that ensued. We urge researchers to pursue the field by assessing the long-term sustainability of the whole-food LCHF approach for overweight children and their families.

\section{Acknowledgements Competing interests}

The authors declare that they have no financial or personal relationships which may have inappropriately influenced them in writing this article.

\section{Authors' contributions}

C.Z. and G.S. were involved in the development of the study design. C.Z., M.W., J.M., D.S. and O.S. were involved in the implementation and data collection. C.Z., M.W. and N.H. conducted the analysis and interpretation of the quantitative data. C.Z. and J.M. were involved with the interpretation of the qualitative data. C.Z. prepared the manuscript. All authors read and approved the final manuscript. 


\section{References}

1. Centres for Disease Control and Prevention. Childhood obesity facts [homepage on the Internet]. Centres for Disease Control and Prevention; 2015 [updated 2015 Aug 27; cited 2016 Nov 14]. Available from: http://www.cdc.gov/healthyschools/ obesity/facts.htm

2. Ministry of Health. Obesity data and stats [homepage on the Internet]. Ministry of Health; 2015 [updated 2015 Dec 2; cited 2016 Nov 14]. Available from: http:// www.health.govt.nz/nz-health-statistics/health-statistics-and-data-sets/obesity data-and-stats

3. Ministry of Health. Childhood obesity plan [homepage on the Internet]. 2016 [cited 2016 Nov 24]. Available from: http://www.health.govt.nz/our-work/ diseases-and-conditions/obesity/childhood-obesity-plan

4. Teicholz $\mathrm{N}$. The scientific report guiding the US dietary guidelines: Is it scientific? BMJ. 2015;351:h4962. https://doi.org/10.1136/bmj.h4962

5. BMJ. The BMJ Press Release. Independent experts find no grounds for retraction of The BMJ article on dietary guidelines [serial online]. BMJ. 2016 [updated 2016 Dec 2; cited 2016 Dec 2]. Available from: http://www.bmj.com/company/wpcontent/uploads/2016/12/the-bmj-US-dietary-correction.pdf

6. Ministry of Health, University of Otago. Methodology Report for the 2008/09 New Zealand Adult Nutrition Survey [homepage on the Internet]. Ministry of Health, University of Otago; 2011 [cited 2016 Dec 2]. Available from: http://www. health.govt.nz/publication/methodology-report-2008-09-nz-adult-nutritionsurvey

7. Taubes $G$. The science of obesity: What do we really know about what makes us fat? An essay by Gary Taubes. BMJ. 2013;346:f1050. https://doi.org/10.1136/bmj. f1050

8. Sackner-Bernstein J, Kanter D, Kaul S. Dietary intervention for overweight and obese adults: Comparison of low-carbohydrate and low-fat diets. A meta-analysis. PLoS One. 2015;10(10). https://doi.org/10.1371/journal.pone.0139817

9. Demol S, Yackobovitch-Gavan M, Shalitin S, Nagelberg N, Gillon-Keren M, Phillip M. Low-carbohydrate (low \& high-fat) versus high-carbohydrate low-fat diets in the treatment of obesity in adolescents. Acta Paediatr. 2009;98(2):346-351. https://doi.org/10.1111/j.1651-2227.2008.01051.x

10. Dunlap BS, Bailes JR, Jr. Unlimited energy, restricted carbohydrate diet improves lipid parameters in obese children. Metab Syndr Relat Disord. 2008;6(1):32-36. https://doi.org/10.1089/met.2007.0023

11. Kirk S, Brehm B, Saelens BE, et al. Role of carbohydrate modification in weight management among obese children: A randomized clinical trial. J Pediatr. 2012;161(2):320.e1-327.e1. https://doi.org/10.1016/j.jpeds.2012.01.041

12. Krebs NF, Gao D, Gralla J, Collins JS, Johnson SL. Efficacy and safety of a high protein, low carbohydrate diet for weight loss in severely obese adolescents. protein, low carbohydrate diet for weight loss in severely obese adolescents.

13. Sondike SB, Copperman N, Jacobson MS. Effects of a low-carbohydrate diet on weight loss and cardiovascular risk factor in overweight adolescents. J Pediatr. 2003;142(3):253-258. https://doi.org/10.1067/mpd.2003.4

14. Gow ML, Ho M, Burrows TL, et al. Impact of dietary macronutrient distribution on $\mathrm{BMI}$ and cardiometabolic outcomes in overweight and obese children and adolescents: A systematic review. Nutr Rev. 2014;72(7):453-470. https://doi. org $/ 10.1111 /$ nure.12111

15. Centres for Disease Control and Prevention. Clinical growth charts [homepage on the Internet]. Atlanta, GA: U.S. Department of Health and Human Services; 2009 [updated 2009 Sep 4; cited 2017 Feb 2]. Available from: https://www.cdc.gov/ growthcharts/clinical_charts.htm

16. Ministry of Health Clinical Trials Research Unit. Clinical guidelines for weight management in New Zealand children and young people [homepage on the Internet]. Wellington; 2009 [cited 2016 Sep 2]. Available from: https://www. health.govt.nz/system/files/documents/publications/weight-managementchildren-guidelines $0 . p d f$
17. Kuczmarski RJ, Flegal KM. Criteria for definition of overweight in transition: Background and recommendations for the United States. Am J Clin Nutr. 2000;72(5):1074-1081. https://doi.org/10.1093/ajcn/72.5.1074

18. Holm S. A simple sequentially rejective multiple test procedure. Scand J Stat. 1979;6(2):65-70.

19. Labtests. Reference intervals [homepage on the Internet]. 2016 [cited 2016 Nov 24]. Available from: https://labtestsonline.org/understanding/analytes/ $\mathrm{hdl} / \mathrm{tab} / \mathrm{test} /$

20. Mascola AJ, Bryson SW, Agras WS. Picky eating during childhood: A longitudinal study to age 11 years. Eat Behav. 2010;11(4):253-257. https://doi.org/10.1016/j. eatbeh.2010.05.006

21. Bailes JR, Strow MT, Werthammer J, McGinnis RA, Elitsur Y. Effect of lowcarbohydrate, unlimited calorie diet on the treatment of childhood obesity: A prospective controlled study. Metab Syndr Relat Disord. 2003;1(3):221-225. https://doi.org/10.1089/154041903322716697

22. Long SJ, Jeffcoat AR, Millward DJ. Effect of habitual dietary-protein intake on appetite and satiety. Appetite. 2000;35(1):79-88. https://doi.org/10.1006/appe. 2000.0332

23. Stubbs RJ, van Wyk MC, Johnstone AM, Harbron CG. Breakfasts high in protein, fat or carbohydrate: Effect on within-day appetite and energy balance. Eur J Clin Nutr. 1996;50(7):409-417.

24. Keskin M, Kurtoglu S, Kendirci M, Atabek ME, Yazici C. Homeostasis model assessment is more reliable than the fasting glucose/insulin ratio and quantitative insulin sensitivity check index for assessing insulin resistance among obese children and adolescents. Pediatrics. 2005;115(4):e500-e503. https://doi. children and adolescents.
org/10.1542/peds.2004-1921

25. Yajnik CS, Katre PA, Joshi SM, et al. Higher glucose, insulin and insulin resistance (HOMA-IR) in childhood predict adverse cardiovascular risk in early adulthood: The Pune Children's Study. Diabetologia. 2015;58:1626-1636. https://doi. org/10.1007/s00125-015-3602-z

26. Roberts CK, Hevener AL, Barnard RJ. Metabolic syndrome and insulin resistance: Underlying causes and modification by exercise training. Compr Physiol. 2013;3(1):1-58. https://doi.org/10.1002/cphy.c110062

27. Quijada Z, Paoli M, Zerpa Y, et al. The triglyceride/HDL-cholesterol ratio as a marker of cardiovascular risk in obese children; association with traditional and emergent risk factors. Pediatr Diabetes. 2008;9(5):464-471. https://doi. org/10.1111/j.1399-5448.2008.00406.x

28. Iwani NA, Jalaludin MY, Zin RM, et al. Triglyceride to HDL-C ratio is associated with insulin resistance in overweight and obese children. Sci Rep. 2017;7:40055 https://doi.org/10.1038/srep40055

29. Pacifico L, Bonci E, Andreoli G, et al. Association of serum triglyceride-to-HDL cholesterol ratio with carotid artery intimamedia thickness, insulin resistance and nonalcoholic fatty liver disease in children and adolescents. Nutr Metab Cardiovasc Dis. 2014;24(7):737-743. https://doi.org/10.1016/j.numecd.2014.01.010

30. Elliot J. Flaws, fallacies and facts: Reviewing the early history of the lipid and diet/ heart hypotheses. Food Nutr Sci. 2014;5:1886-1903. https://doi.org/10.4236/ fns.2014.519201

31. Ravnskov U, Diamond DM, Hama R, et al. Lack of an association or an inverse association between low-density-lipoprotein cholesterol and mortality in the elderly: A systematic review. BMJ Open. 2016;6(6):e010401. https://doi.org/ 10.1136/bmjopen-2015-010401

32. Rizzo M, Berneis K. Low-density lipoprotein size and cardiovascular risk assessment. QJM. 2006;99(1):1-14. https://doi.org/10.1093/qjmed/hci154

33. Toth PP. High-density lipoprotein and cardiovascular risk. Circulation. 2004 109(15):1809-1812. https://doi.org/10.1161/01.CIR.0000126889.97626.B8

34. Crofts CAP, Wheldon MC, Zinn C, Lan-Pidhainy X, Wolever TMS, Schofield G. Assessing the test-retest repeatability of insulin resistance measures: Homeostasis model assessment 2 and oral glucose insulin sensitivity. J Insulin Resist. 2017;2(1):a27. https://doi.org/10.4102/jir.v2i1.27 


\section{Appendix 1: Telephone protocol}

\section{General questions}

(1) How is everything going in general on this diet? What's working well? What's not working well?

(2) How is your child feeling overall?

(3) Can I help you with anything that is not working well?

(4) Can I help with any queries you might have about any aspect of the diet?

(5) FOR SUBSEQUENT PHONE CONVERSATIONS: The last time we talked, you found [issue] to be difficult. How has that been this week?

(6) (if necessary) What might need to be changed in order to help you follow the new way of eating?

(7) (if necessary) What are your ideas about making these changes?

\section{4-hour recall}

Can you take me through yesterday's food intake for your child?

Breakfast

Lunch

Dinner

Other

\section{Food frequency questionnaire (11 questions)}

(1) In the past 7 days, on how many occasions did you consume bread/toast/ wraps/bread rolls? (Interviewer to encircle the answer)

$\begin{array}{llllllllllll}0 & 1 & 2 & 3 & 4 & 5 & 6 & 7 & 8 & 9 & 10 & >10\end{array}$

(2) In the past 7 days, on how many occasions did you consume pasta or noodles? This includes all pasta dishes, lasagne and noodles such as 2 min noodles, Vietnamese noodles, etc. (Interviewer to encircle the answer)

$\begin{array}{llllllllllll}0 & 1 & 2 & 3 & 4 & 5 & 6 & 7 & 8 & 9 & 10 & >10\end{array}$

(3) On how many occasions over the past 7 days did you consume rice? This includes brown or white rice and sushi. (Interviewer to encircle the answer)

$\begin{array}{llllllllllll}0 & 1 & 2 & 3 & 4 & 5 & 6 & 7 & 8 & 9 & 10 & >10\end{array}$

(4) On how many occasions over the past 7 days did you eat fast food or takeaways from places like McDonalds or Burger King? (Interviewer to encircle the answer)
$0 \quad 1$
3
45
$\begin{array}{lllll}7 & 8 & 9 & 10 & >10\end{array}$

(5) On how many occasions over the past 7 days have you consumed crackers, or snacks such as crackers, potato chips, corn chips and pies? (Interviewer to encircle the answer)

$\begin{array}{llllllllllll}0 & 1 & 2 & 3 & 4 & 5 & 6 & 7 & 8 & 9 & 10 & >10\end{array}$

(6) On how many occasions over the past 7 days did you drink fruit juices, soft drinks, sports drinks or energy drinks? Do not include diet varieties.

(Interviewer to encircle the answer)

$\begin{array}{llllllllllll}\text { vii } & 1 & 2 & 3 & 4 & 5 & 6 & 7 & 8 & 9 & 10 & >10\end{array}$

(7) On how many occasions over the past 7 days did you consume lollies, sweets, chocolates or confectionary? (Interviewer to encircle the answer)

$\begin{array}{llllllllllll}0 & 1 & 2 & 3 & 4 & 5 & 6 & 7 & 8 & 9 & 10 & >10\end{array}$

(8) On how many occasions over the past 7 days did you consume baked goods such as manufactured or homemade biscuits or cakes? (Interviewer to encircle the answer)
45
(1)
$\begin{array}{lllll}7 & 8 & 9 & 10 & >10\end{array}$ 


\section{Appendix 2: Focus group questions}

\section{Adults}

1. How easy or difficult was it for you / your child to stick to eating in such a way for the duration of the study? (perhaps ask for an overall rating from everyone?)

2. What were the factors that made it easy?

3. What were the factors that made it difficult / barriers?

4. Were there any meal or snack times / occasions that you found particularly easy/difficult to follow the new way of eating? (i.e. school holidays etc.)

5. What would make it easier for your child to stick to this way of eating?

6. Can you comment on whether you and / or your child enjoyed eating this way and the reasons for this.

7. How did this way of eating fit in with your family life? Ask who else in the family follow this

8. How did this way of eating fit in with your child's school / social life?

9. Do you think your child (and you) will continue with this way of eating?

10. Why / why not?

11. Can you describe how eating like this affected your budget? (asked separately and in the focus group)

\section{Children}

1. How easy or hard was it for you to stick to eating like this?

2. What was easy about it? / When were the easy times?

3. What was hard about it? / When were the hard times?

4. How did other kids at school react? (i.e. tell me about what (if anything) was different in the school environment?)

5. What would make it easier for you to stick to this way of eating?

6. Did you enjoy eating this way? Why? / Why not?

7. Which foods did you like the most?

8. Which foods did you like the least?

9. How did this way of eating fit in with your friends at school / family life?

10. Do you think you will carry on eating like this?

11. Why / why not? 